\title{
Pattern recognition of high-resolution computer tomography (HRCT) chest to guide clinical management in patients with mild to moderate COVID-19
}

\section{Bavaharan Rajalingam, Ethirajan Narayanan1, Praveen Nirmalan², Kamalanthan Muthukrishnan³,

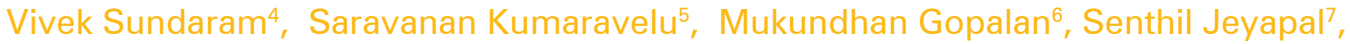 Baskaran Rajalingam, Vijay Khanna ${ }^{9}$, Praveen Dhoss ${ }^{10}$, Gopinath ${ }^{11}$}

DNB,Managing Director, Magnum Imaging \& Diagnostics, Trichy, ${ }^{1}$ Retired Professor, Social \& Preventive Medicine, Chidambaram, Trichy, Tamil Nadu, ${ }^{2} \mathrm{MPH}$, Reasearch Head, Amma Education Research Foundation, Kochi, ${ }^{3}$ DNB, Consultant, Magnum Imaging \& Diagnostics, ${ }^{4}$ Sundaram Hospital, Director, ${ }^{5}$ Director, Saravana Medical Centre, ${ }^{6}$ ABC Hopsital, Director, ${ }^{7}$ Director, GVN Hopsital, Singarathope, ${ }^{8}$ Consultant Physician, GVN Hopsital, Singarathope, ${ }^{9}$ Consultant Critcal Care, Retna Global Hopsital, ${ }^{10}$ Director, Retna Global Hopsital, ${ }^{11}$ Emergency Medical Officer, Sundaram Hospital, Trichy, Tamil Nadu, India

Correspondence: Dr. Bavaharan Rajalingam, Magnum Imaging \& Diagnostics, 9/10 Ramachandrapuram, $11^{\text {th }}$ Cross East Thillainagar, Thennur, Trichy - 620 017, Tamil Nadu, India. E-mail: bavaharan@gmail.com

\begin{abstract}
Aim: To describe the distribution of lung patterns determined by High Resolution Computed Tomography (HRCT) in COVID patients with mild and moderate lung involvement and outcomes after early identification and management with steroids and anticoagulants. Material and Methods: A cross sectional study of COVID-19 patients with mild and moderate lung involvement presenting at 5 healthcare centres in Trichy district of South TamilNadu in India. Patients underwent HRCT to assess patterns and severity of lung involvement, Inflammatory markers (LDH/Ferritin) and D-Dimer assay and clinical correlation with signs and symptoms. Patients were assessed for oxygen, steroid and anticoagulant therapy, clinical recovery or progression on follow up and details on mortality were collected. The RSNA, Fleischer Society guidelines and CORADS score was used for radiological reporting. New potential classification of patterns of percentage of lung parenchyma involvement in Covid patients is being suggested. Results: The study included 7,340 patients with suspected COVID and 3,963 (53.9\%) patients had lung involvement based on HRCT. RT PCR was positive in $74.1 \%$ of the CT Positive cases. Crazy Pavement pattern was predominant ( $n=2022,51.0 \%)$ and Ground Glass Opacity (GGO) was found in 1,941 (49.0\%) patients in the study. Severe lung involvement was more common in the Crazy Pavement pattern. Patients with GGO in moderate lung involvement were significantly more likely to recover faster compared to Crazy Pavement pattern $(P$ value $<0.001)$. Conclusion: HRCT chest and assessment of lung patterns can help triage patients to home quarantine and hospital admission. Early initiation of steroids and anticoagulants based on lung patterns can prevent progression to more severe stages and aid early recovery. HRCT can play a major role to triage and guide management especially as RT PCR testing and results are delayed for the benefit of patients and in a social cause to decrease the spread of the virus
\end{abstract}

Key words: COVID 19; crazy pavement pattern; ground glass opacity; HRCT

\begin{tabular}{|l|l|}
\hline \multicolumn{2}{|c|}{ Access this article online } \\
\hline Quick Response Code: & \\
\hline & Website: \\
\hline & www.iji.org \\
\cline { 2 - 3 } & DOI: \\
\hline
\end{tabular}

This is an open access journal, and articles are distributed under the terms of the Creative Commons Attribution-NonCommercial-ShareAlike 4.0 License, which allows others to remix, tweak, and build upon the work non-commercially, as long as appropriate credit is given and the new creations are licensed under the identical terms.

For reprints contact: WKHLRPMedknow_reprints@wolterskluwer.com

Cite this article as: Rajalingam B, Narayanan E, Nirmalan P, Muthukrishnan K, Sundaram $\mathrm{V}$, Kumaravelu $\mathrm{S}$, et al. Pattern recognition of high-resolution computer tomography (HRCT) chest to guide clinical management in patients with mild to moderate COVID-19. Indian J Radiol Imaging 2021;31:S110-8.

Received: $18-S e p-2020$

Revised: 14-Dec-2020

Accepted: 21-Dec-2020 Published: 23-Jan-2021 


\section{Introduction}

Globally, high resolution computed tomography (HRCT) chest is currently underutilized for the evaluation and management of patients affected with COVID-19. HRCT is used in many developed countries for severe cases of COVID pneumonia primarily to assess the status of the lung and not in the clinical context of treating the patients. Pulmonary damage is the most important causes of morbidity and mortality globally although SARS CoV-2 has extra pulmonary manifestations including sepsis syndrome.

The high sensitivity of HRCT in patients infected by SARS-CoV-2 makes it suitable for inclusion in clinical management protocols ${ }^{[1]}$ The majority of patents with COVID 19 are in the mild to moderate spectrum. The clinical management of these patients, who may be symptomatic or asymptomatic, is important from the clinical perspective of the individual patient as well as from a public health perspective of breaking or controlling the potential transmission of the virus. HRCT chest can help to guide clinical management in mild \& moderate cases of COVID pneumonia, provide useful information to triage for home quarantine or hospital admissions and prevent the worsening of patients to more severe states.

In this article, we describe the patterns of lung involvement, using HRCT, in patients, categorized as mild to moderate to severe. The outcomes of early initiation of steroid and anticoagulant administration in HRCT positive mild and moderate cases with Inflammatory markers and D-Dimer elevation including reduction of transitions to more severe stages and hospital stay and costs for patients with moderate lung involvement are presented.

\section{Material and Methods}

A cross sectional study design was conducted from May 152020 to August 30 2020, at five healthcare centers in Trichy district of South Tamilnadu in India. The study centers included Magnum Imaging \& Diagnostics, which was responsible for the imaging and diagnostic testing of COVID suspected patients, and ABC hospital, GVN Hospital Singarathope, Sundaram Hospital, Saravana Hospital \& Retna Global Hospital, which were responsible for the clinical care management of patients with COVID-19.

Patients aged 10 to 70 years, suspected to have COVID related symptoms and exposure to infected persons were included in the study. Patients with pre-surgical conditions, Diabetes, Hypertension and Renal Failure who were referred for radiology examinations and were found to have findings suggestive of COVID-19 on examination, were also included in the study.COVID-related symptoms were considered to include one or more of the following viz., fever, sore throat, body pain, diarrhea and breathlessness. Patients with pre-existing interstitial lung disease, unilateral destroyed lung post Tuberculosis, previous history of heart diseases, previous history of asthma, known primary lung malignancy or lung metastatic malignancies, readmissions, patients with $>60 \%$ lung involvement, irrespective of lung involvement pattern, pleural effusion or pneumothorax or pneumomediastinum identified in HRCT were excluded from the study. Patients who were lost to follow up were also excluded from the study.

Every patient underwent evaluation with HRCT chest, Inflammatory markers (LDH/Ferritin) and D-Dimer assay. The diagnostic test findings were correlated with clinical staging by $\mathrm{SaO} 2$ in room air and the physician's decision to admit the patient. On follow up, patients were assessed for oxygen, steroid and anticoagulant therapy, clinical recovery or progression. Details on mortality were also collected. The post recovery $\mathrm{CT}$ chest or progression to fibrosis or sequelae were not included in the scope of this study.

\section{CT protocol}

Multidetector CT scanners (GE128 slice \& Siemens 16 slice) were used for the examinations. Scanning parameters were identical to the manufacturer's standard recommended pre-setting for a thorax routine. Images were reconstructed with a $0.625 \mathrm{~mm}$ slice thickness in all cases using the classic filtered back-projection method with a soft tissue kernel of B20 and a lung kernel of B60. Coronal and sagittal multiplanar reconstructions were also available in all cases.

Implementation of appropriate infection prevention and control measures, for the healthcare workers and the CT room, were arranged in all suspected COVID cases undergoing CT and included prompt sanitation of CT facility and patient's isolation. Appropriate, recommended social distancing guidelines were followed and included minimal waiting times and dedicated patient routes.

\section{Image analysis}

Definitions of radiological terms like ground glass opacity (GGO), crazy-paving pattern, and pulmonary consolidation were based on the standard glossary for thoracic imaging reported by the Fleischner Society. ${ }^{[2]}$ Based on two separate publications by Hansell \& Salehi, ${ }^{[3,4]}$ the diagnosis of a suspected SARS-CoV-2 pneumonia was established considering the following chest CT patterns: GGO, crazy-paving, and consolidation and the diagnostic confidence of COVID lung was determined based on CORADS. ${ }^{[5]}$

Once the CT was classified as CORADS 4 or 5 (High or Very High Suspicion), Radiological Severity Classification 

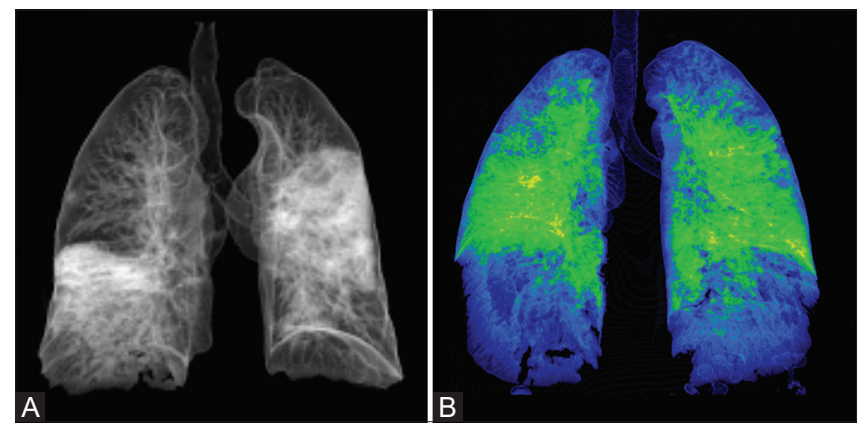

Figure 1 ( $A$ and $B$ ): $A$ and $B: 3 D$ segmentation Volume images showing Mild and Moderate lung involvement, for segmentation and percentage analysis with the software
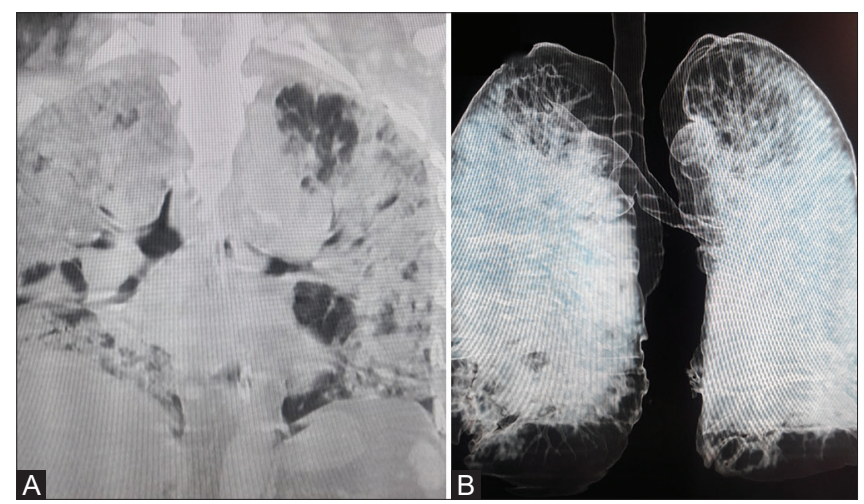

Figure 3 (A and B): A and B: Severe Disease by coronal CT \& Segmentation analysis A 62 year-old male $75 \%$ lung involvement - "M" pattern, hospitalised with breathlessness $\mathrm{SaO} 285 \%$ in room air with elevated inflammatory markers and D-Dimer

was made on the percentage of Lung Involvement (Using Automated software) into mild, moderate and severe, which is $<30 \%, 30-60 \%$ and $>60 \%$, respectively [Figures $1-3$ ].

We evaluated the pattern of percentage lung involvement, which we could assess by HRCT chest, along with D-Dimer \& inflammatory markers (LDH \& Ferritin) to predict the possibility of worsening of the disease condition, as the important clinical problems were the severity of inflammation, rhabdomyolysis and thrombogenesis.

The HRCT Lung in COVID cases were reported with mention of predominant pattern, viz., " $\mathrm{G}$ ", when there was predominant Ground glass opacities, " $\mathrm{C}$ ", when there was predominant Crazy-paving or consolidation \& " $\mathrm{M}$ " for mixed pattern or those pattern that could not be properly evaluated or categorized as only G or C. For example, if there is $30-60 \%$ lung involvement with predominant crazy-paving pattern, it was reported as "CORADS 5 - Moderate Disease -C" [Figure 4A and B]

Informed consent is routinely obtained from every patient presenting for diagnostic imaging, lab tests and clinical assessments at the study centers. Data were entered into a spreadsheet and analyzed further with respect to the main outcomes. A Chi-square test was used to determine

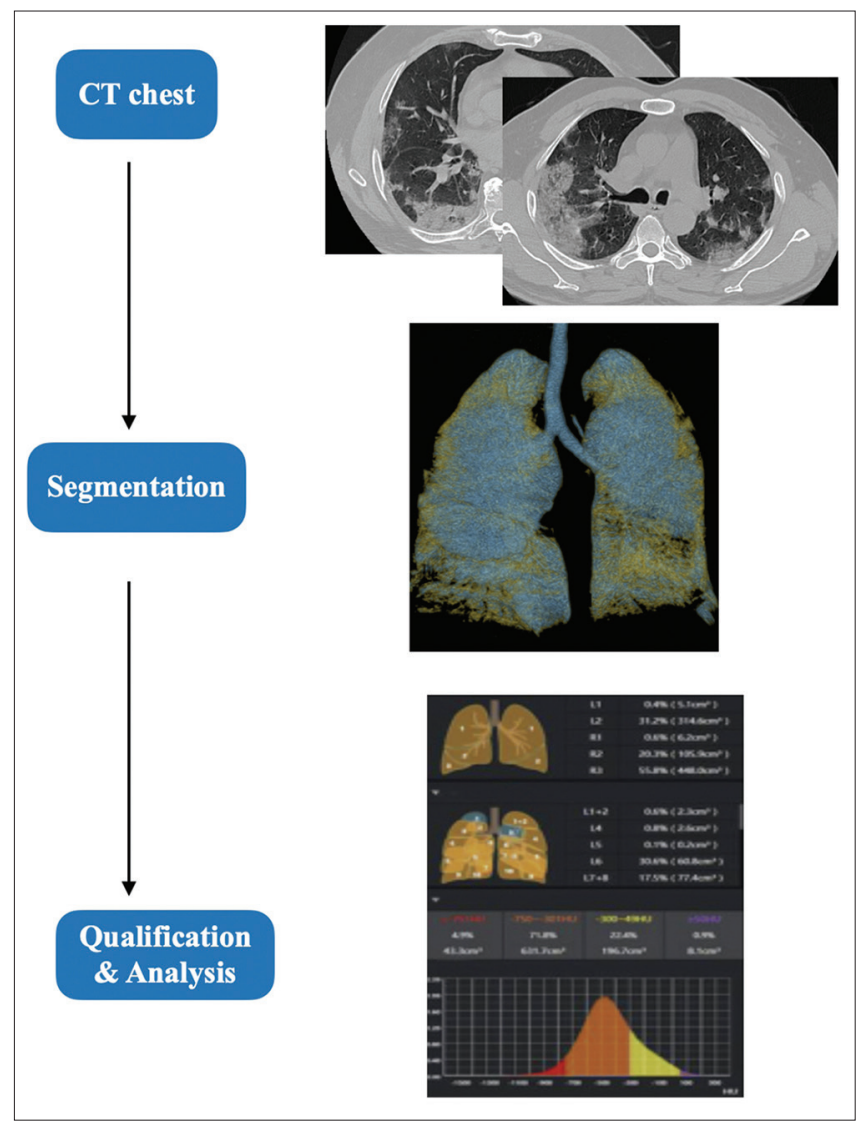

Figure 2: Volume of lung involvement by segmentation, Qualification and Analysis
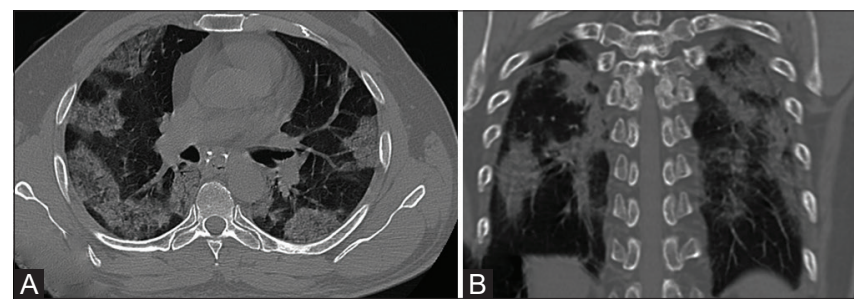

Figure 4 (A and B): A and B: Axial and Coronal - Crazy paving pattern - moderate disease (CORADS -5 - Moderate Disease -"C" Pattern

statistical significance of important associations and a $P$ value $<0.05$ was considered as statistically significant.

\section{Results Analysis}

The study included 7,340 patients with suspected COVID and 3,963 (53.9\%) patients had lung involvement based on HRCT. The majority of these 3,963 subjects were aged 30 to 50 years $(n=-1741,43.93 \%)$ and $2241(56.55 \%)$ were males. Among the 3,963 patients with a positive HRCT, Mild Lung involvement was identified in 1,783 (43\%), moderate lung involvement in 1,268 (32\%) and severe lung involvement in 912 (25\%). Table 1 shows the RT PCR status in these 3,963 patients. 
The predominant HRCT pattern in this population was Crazy Pavement pattern $(n=2022,51.0 \%)$ and GGO was found in 1,941 (49.0\%) patients in the study. Severe lung involvement was more common in the Crazy Pavement pattern of lung involvement [Table 2] Patients with GGO and moderate lung involvement were significantly more likely to recover faster compared to patients with Crazy Pavement pattern (Chi-square test, $P$ value $<0.001$ ) [Table 3].

Elevated Inflammatory Markers (LDH/ferritin/D-Dimer) were found in $134(17.3 \%)$ of patients with GGO and moderate lung involvement and $488(87.6 \%)$ of patients with Crazy Pavement pattern (Chi square $P$ value $<0.001$ ). All these patients were followed up with HRCT after 3 days and showed increase in Septal thickening/Band atelectasis/Plate atelectasis. All these patients were started on Anticoagulant \& Steroids and HFNO was started in cases of Hypoxemia. Mortality in Moderate Lung Involvement (Any Pattern total patients 1263) was $18(1.4 \%)$ irrespective of treatment with Steroids \& Anticoagulants. All these patients had pre-existing conditions such as Diabetes, Hypertension or renal failure. Patients sowing ground glass opacity with mild and moderate lung involvement by HRCT, were significantly less likely to need hospitalization compared to those with Crazy Pavement Pattern (Chi square test, $P<0.001$ ) [Table 4].

\section{Discussion}

On February 11, 2020, the WHO Director-General, Dr. Tedros Adhanom Ghebreyesus, announced that the disease caused by this new CoV was a "COVID-19," which is the acronym of "coronavirus disease 2019". ${ }^{[6]}$ This new virus initially reported from Wuhan district of China in 2019 is very contagious and has quickly spread globally. In a meeting on January 30, 2020, per the International Health Regulations (IHR, 2005), the outbreak was declared by the WHO a Public Health Emergency of International Concern (PHEIC) as it had spread to 18 countries with four countries reporting human-to-human transmission. An additional landmark occurred on February 26, 2020, as the first case of the disease, not imported from China, was recorded in the United States (US). ${ }^{[6]}$ After which, COVID-19 quickly crossed the ocean and as of September 15,2020 , about 4.8 million cases with 81,000 deaths has been recorded in India, whereas about 29.2 million with 926,000 deaths has been recorded throughout the world. Although over time the lethality rate (total number of deaths for a given disease in relation to the total number of patients) of COVID-19 has been significantly lower than that of the SARS and MERS epidemics, the transmission of the SARS-CoV-2 virus is much larger than that of the previous viruses, with a much higher total number of deaths. It has been estimated that about one in five individuals
Table 1: RT PCR status in the 3,963 patients with identifiable lung involvement in HRCT

\begin{tabular}{lc}
\hline RT PCR status & CT Positive \\
\hline Positive & $2,940(74.1 \%)$ \\
Negative & $1,023(25.9 \%)$ \\
\hline
\end{tabular}

Table 2: Pattern of lung involvement by severity of lung of involvement

\begin{tabular}{lcc}
\hline $\begin{array}{l}\text { Lung } \\
\text { Involvement }\end{array}$ & $\begin{array}{c}\text { Ground Glass } \\
\text { Opacity } n, \%\end{array}$ & $\begin{array}{c}\text { Crazy Pavement } \\
\text { Pattern } n, \%\end{array}$ \\
\hline Mild & $963,49.61 \%$ & $821,40.60 \%$ \\
Moderate & $712,31.68 \%$ & $557,27.54 \%$ \\
Severe & $266,13.70 \%$ & $644,31.84 \%$ \\
\hline
\end{tabular}

Table 3: Recovery rate by lung pattern involvement in COVID patients with moderate lung involvement

\begin{tabular}{lcc}
\hline & $\begin{array}{c}\text { Ground glass } \\
\text { Opacity } \\
n=712\end{array}$ & $\begin{array}{c}\text { Crazy } \\
\text { Pavement } \\
\text { pattern } n=-557\end{array}$ \\
\hline Asymptomatic and recovered & $510(71.63 \%)$ & $131(23.52 \%)$ \\
Became symptomatic during course of illness & $202(28.37 \%)$ & $426(76.48 \%)$ \\
\hline
\end{tabular}

Table 4: Hospitalization by lung pattern in COVID patients with moderate lung involvement

\begin{tabular}{lcc}
\hline & $\begin{array}{c}\text { Ground Glass } \\
\text { Opacity } n=712\end{array}$ & $\begin{array}{c}\text { Crazy Pavement } \\
\text { Pattern } n=-557\end{array}$ \\
\hline Hospitalized & $109(15.31 \%)$ & $488(87.61 \%)$ \\
Did not need hospitalization & $603(84.69 \%)$ & $69(12.39 \%)$ \\
\hline
\end{tabular}

worldwide could be at increased risk of severe COVID-19 disease if they become infected, due to underlying health conditions. ${ }^{[7]}$

CoVs are enveloped, positive-stranded RNA viruses with nucleocapsid the SARS-CoV-2 virus, like the closely related MERS and SARS coronaviruses, effects its cellular entry via attachment of its virion spike protein (S protein) to the angiotensin-converting enzyme 2 (ACE2) receptor, which is expressed in the acini. The spike RBD (Receptor-Binding Domain) allows the binding to the ACE2 receptor in the lungs and other tissues. The presence within the spike protein of an amino acid site (polybasic site) allows the functional processing of the same by the human enzyme furin (protease). This process allows the exposure of the fusion sequences and therefore the fusion of the viral and cell membranes, a necessary passage for the virus to enter the cell. ${ }^{[6]}$ The data so far available seem to indicate that the viral infection is capable of producing an excessive immune reaction in the host. In some cases, a reaction takes place which as a whole is labeled a 'cytokine storm'. The effect is extensive tissue damage with dysfunctional coagulation. The Italian researchers introduced the term of MicroCLOTS (microvascular COVID-19 lung vessels obstructive thromboinflammatory syndrome) for underlying the lung viral injury associated 

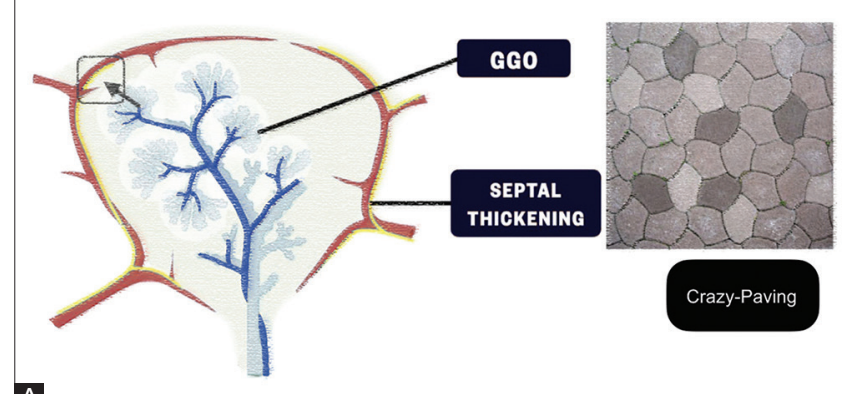

A

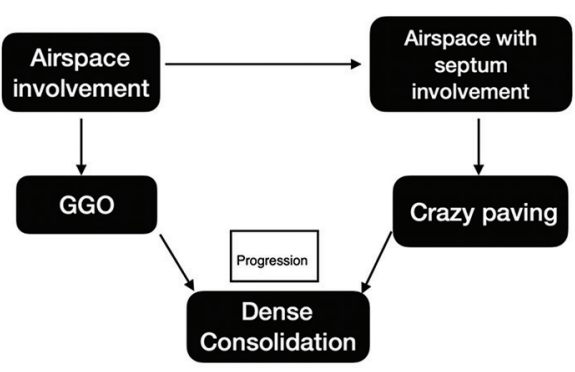

B

Figure 5 ( $A$ and $B$ ): A and $B$ Anatomy of the Lobule of Lung and Pattern of Lung involvement: Secondary lobule is made up of Centrilobular structures, Lobular parenchyma and the septal structures. Secondary pulmonary lobules represent a cluster of up to 30 acini $^{[9]}$ (Light blue) supplied by a common distal pulmonary artery (blue) and bronchiole. ${ }^{[10-12]}$ These clustered acini are bounded by interstitial fibrous septa (interlobular septa), which has lymphatics (yellow) and Pulmonary veins (red) which outline an irregular polyhedron of varying size between 1 and $2.5 \mathrm{~cm}$. Peripheral lobules are larger and cuboidal/ pyramidal, while central lobules tend to be smaller and hexagona[112] When the airspace is involved the HRCT reveals ground glass opacities (GGO), with dilated vessel sign and when the inflammation progresses through the lobular parenchyma and involves the septum, crazy-paving pattern is seen, which is due to interstitial vasculitis involving the pulmonary vein (box with arrow)

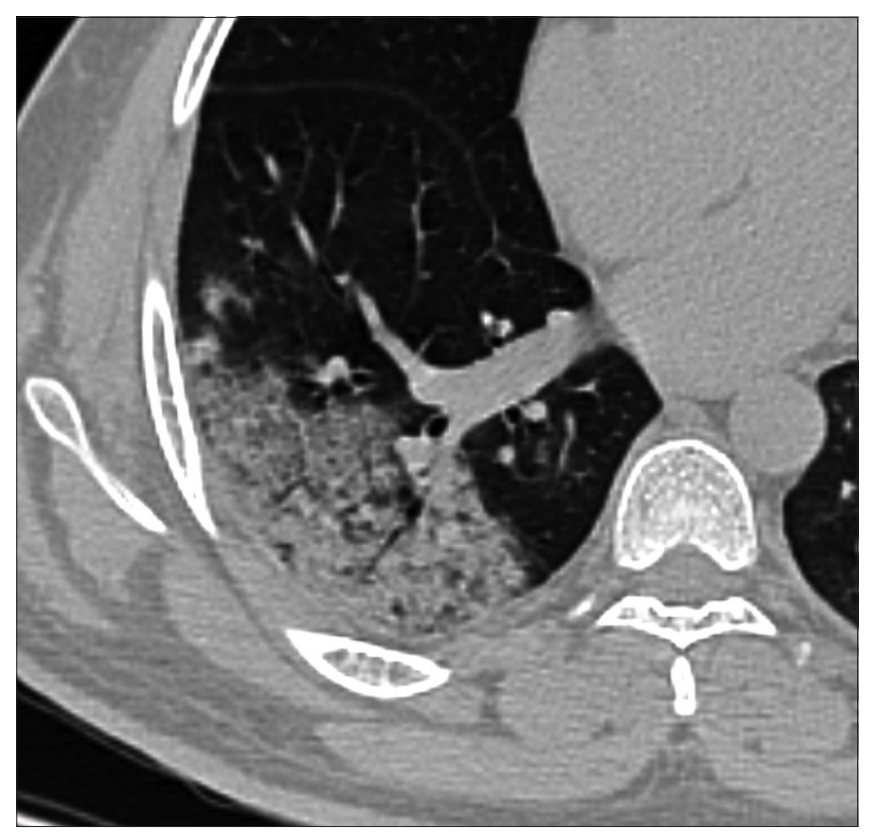

Figure 7: Crazy Paving and areas of consolidation

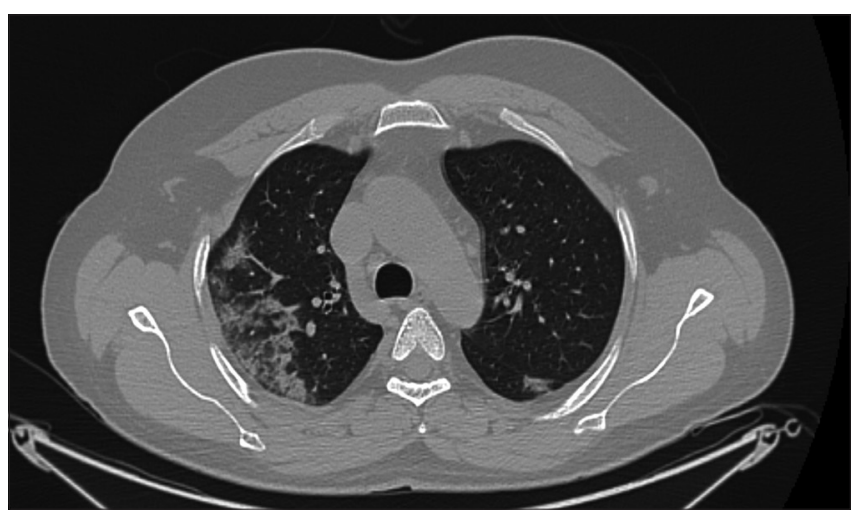

Figure 6: Ground glass opacities in posterior segments of bilateral upper lobes with dilated terminal pulmonary artery (blue double head arrow) - (due to airspace inflammation - Lung response to the Viral disease)

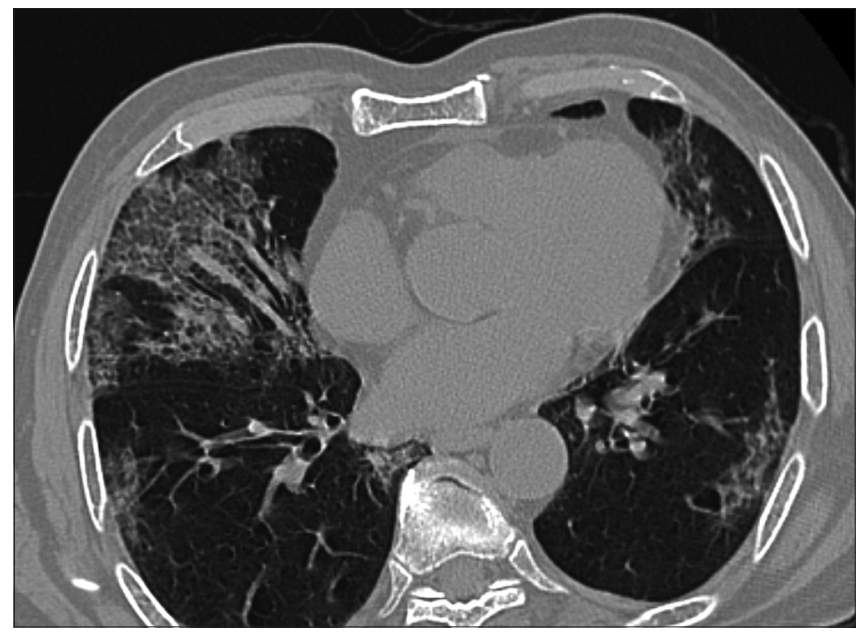

Figure 8: Crazy paving pattern

with the inflammatory reaction and the microvascular pulmonary thrombosis. ${ }^{[8]}$

The Lung changes are host response to the virus attachment in the airspace. These changes are predominantly seen between 5 to 7 days on HRCT. So, it is prudent to do CT chest between day 5 to 7 from the onset of symptoms, which is the intermediate phase between late viral disease and early host response. Doing a HRCT chest earlier may give false sense of wellbeing and a false negative result. But in cases of Suspected Covid, with preexisting lung disease (COPD/ILD) or prior oxygen dependency or hypoxia during the $1^{\text {st }}$ visit to hospital, early HRCT may be indicated, to assess the status of underlying Lung damage.

The knowledge of basic structural and functional unit [Figure 5A], is essential in understanding the pathophysiology of inflammatory cascade that happens in the lung parenchyma which is the underlying cause for the clinical deterioration of the affected patients. Secondary pulmonary lobules represent a cluster of up 
to 30 acini $^{[9]}$ supplied by a common distal pulmonary artery and bronchiole. ${ }^{[10-12]}$ These clustered acini are bounded by interstitial fibrous septa (interlobular septa) which outline an irregular polyhedron of varying size between 1 and $2.5 \mathrm{~cm}^{2}$ Peripheral lobules are larger and cuboidal/pyramidal, while central lobules tend to be smaller and hexagonal. ${ }^{[12]}$ When the airspace is involved by the disease process due to ACE expression in the acini, the HRCT reveals ground glass opacities (GGO), where there is associated terminal pulmonary arteritis, giving the dilated vessel sign [Figure 6]. When the inflammation progresses through the lobular parenchyma \& involves the septum, crazy-paving pattern is seen, which is due to involvement of Pulmonary veins, as a part of endothelial dysfunction \& the Lymphatic involvement [Figures 7 and 8]. These patients showing predominantly septal thickening involving 30 to $60 \%$ lung parenchyma shows elevated inflammatory markers \& D-Dimer level [Chart 1] The interstitial vasculitis involving the pulmonary veins hampers the oxygen transfer to the systemic circulation, how much ever oxygen is administered, through that disease affected lobule. So, these patients were benefitted by Steroid \& Anticoagulant administration, thereby reducing the thrombo-inflammation and effectively managing the patients by facilitating the transfer of oxygen administered, in cases of hypoxia, into the systemic circulation.

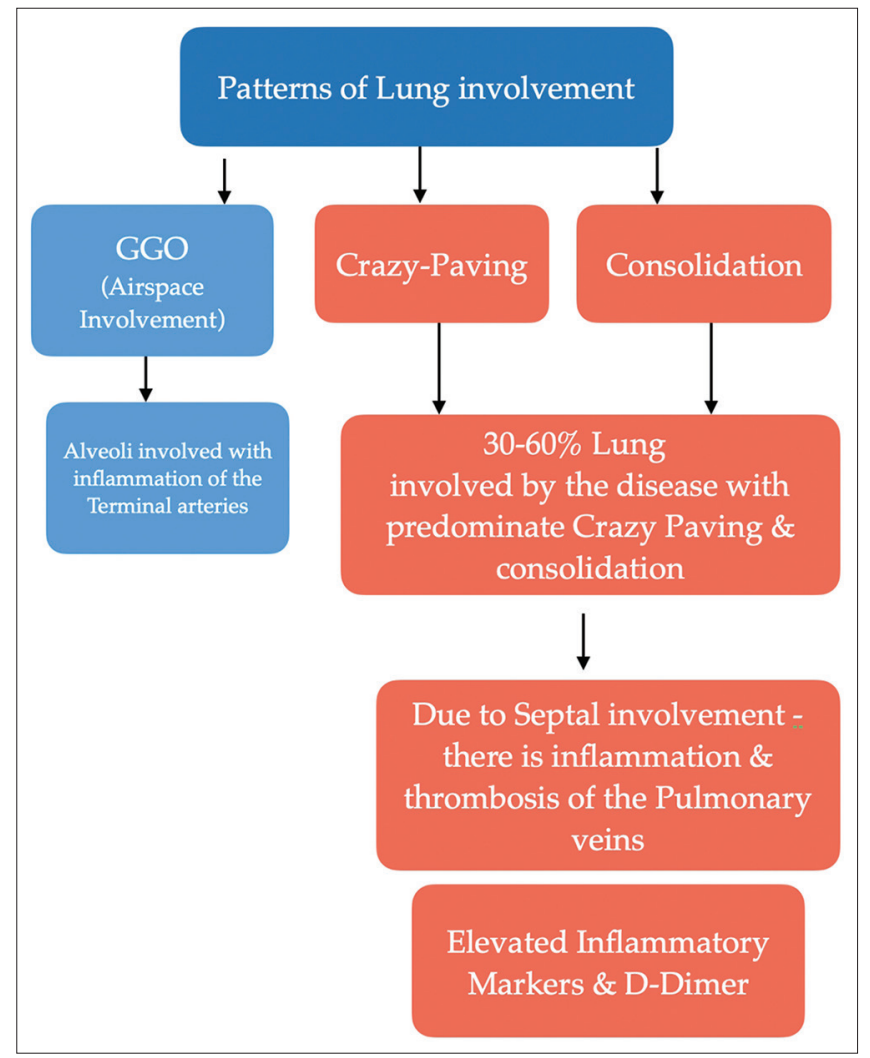

Chart 1: Pattens of Lung involvement, its pathophysiology and correlation with Biochemical parameters
This is also supported by Tian et al. ${ }^{[13]}$ who reported histopathological data obtained on the lungs of two patients who underwent lung lobectomies for adenocarcinoma. These patients were retrospectively found to have had the infection at the time of surgery in which the authors reported vascular congestion combined with inflammatory clusters of fibrinoid material and multinucleated giant cells and hyperplasia of pneumocytes. Zhang et al. ${ }^{[14]}$ performed a postmortem transthoracic needle lung biopsy in a patient who died of COVID-19. Immunostaining showed diffuse alveolar injury and an important alveolar expression of viral antigens and the authors ${ }^{[15]}$ offered a detailed picture of the histological patterns in lung and extrapulmonary tissues. characterized by capillary congestion, necrosis of pneumocytes, hyaline membrane, interstitial edema, pneumocyte hyperplasia, reactive atypia and Platelet-fibrin thrombi in small vessels were the expression of intravascular coagulopathy with findings suggestive for vascular dysfunction, in lung and other tissues.

Since the veins in the septa are obliterated and no metabolite or oxygen transfer in the capillary level, there is not the lymph exudation, which is the possible reason why inspire of so much of lung inflammation, Pleural effusion is not the main feature in Covid Pneumonia, unless there is myocarditis or coronary disease. Since the air pressure in the Alveoli is less than the pleural pressure when the inflammation resolves, band atelectasis is the common feature in the post infective sequel.

The Dutch Radiological Society developed CO-RADS based on other efforts for standardization, such as the Lung Imaging Reporting and Data System or Breast Imaging Reporting and Data System. CO-RADS assesses the suspicion for pulmonary involvement of COVID-19 on a scale from 1 (very low suspicion) to 5 (very high suspicion). The system is meant to be used in patients with moderate to severe symptoms of COVID-19. The system was evaluated by using 105 chest CT scans of patients admitted to the hospital with clinical suspicion of COVID-19 and in whom reverse transcription-polymerase chain reaction (RT-PCR) was performed. ${ }^{[5]}$ This system gives the diagnostic confidence in Covid lung. However, it does not provide insight to the pattern of lung involvement or the volume of lung involved by the disease.

The Radiological Society of North America (RSNA) has released a consensus statement endorsed by the Society of Thoracic Radiology and the American College of Radiology (ACR) that classifies the CT appearance of COVID-19 into four categories for standardized reporting language. ${ }^{[16]}$ The typical appearances include peripheral, bilateral, GGO +/- consolidation or visible intralobular lines ("crazy paving" pattern), multifocal GGO of rounded morphology $+/$ - consolidation or visible intralobular lines ("crazy paving" pattern) and reverse halo sign or 
other findings of organizing pneumonia. The indeterminate appearances include absence of typical CT findings and the presence of multifocal, diffuse, perihilar, or unilateral GGO +/- consolidation lacking a specific distribution and are non-rounded or non-peripheral few very small GGO with a non-rounded and non-peripheral distribution. Third, the atypical appearances include absence of typical or indeterminate features and the presence of isolated lobar or segmental consolidation without GGO discrete small nodules (e.g., centrilobular, tree-in-bud), lung cavitation and smoother interlobular septal thickening with pleural effusion and finally, negative for pneumonia where there is no CT features to suggest pneumonia, in particular, absent GGO and consolidation. ${ }^{[16]}$

The segmental and lobar involvement of lung by Covid is given by CT severity score. CT severity score ${ }^{[17]}$ (total score out of 25) does not correlate with the biochemical parameters and clinical severity in cases of $<60 \%$ lung involvement, which takes into account only the percentage of segmental involvement rather than the pattern of disease process. With Severity score alone, it is unclear, which of these subsets will fare well or worsen during the course of illness and also there is no clear correlation with the biochemical worsening. We also cannot ascertain how to triage these patients to home quarantine or close monitoring or to hospitalize with general CT severity score alone. Clinically patients

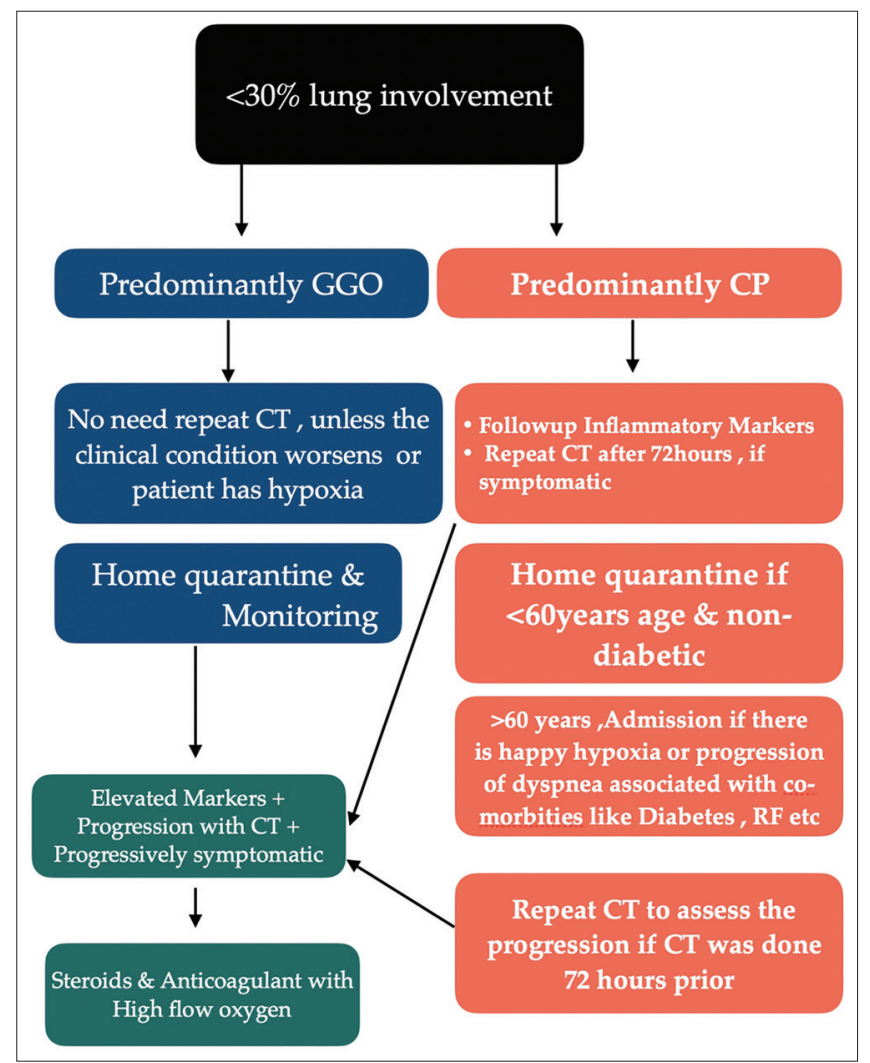

Chart 2: Patterns in cases with Less than $30 \%$ Lung involvement on HRCT by Covid-19 with investigation and management protocol are classified as mild, moderate, and severe by oxygen saturation in room air. Radiological Classification has to be made on the percentage of Lung Involvement (Preferably using Automated software) into mild, moderate, and severe, which is $<30 \%, 30-60 \%$, and $>60 \%$, respectively with special mention of predominant pattern $\mathrm{G} / \mathrm{C} / \mathrm{M}$, as explained earlier in this article. Patients with more than $60 \%$ lung involvement by HRCT, usually have mixed pattern on CT and are predominantly symptomatic and shows elevated inflammatory markers invariably, who needs hospitalization and different management plan, including Plasma therapy, Immunomodulators, Free-Radical scavengers or Ventilation according to the clinical and biochemical parameters.

The major morbidity in this cross-sectional population of Trichy District, South India are the mild and moderate group, which is attributed to the strain of virus and tropical climate. When HRCT shows ' $\mathrm{C}$ " or " $\mathrm{M}$ " pattern, biochemical investigations (Inflammatory markers and D-Dimer) were ordered and the treatment was initiated based on the biochemical and clinical parameters. In these cases, HRCT gives us the insight on the possible pathology, used in guiding these patients for further need for investigations and appropriate management [Charts 2 and 3].

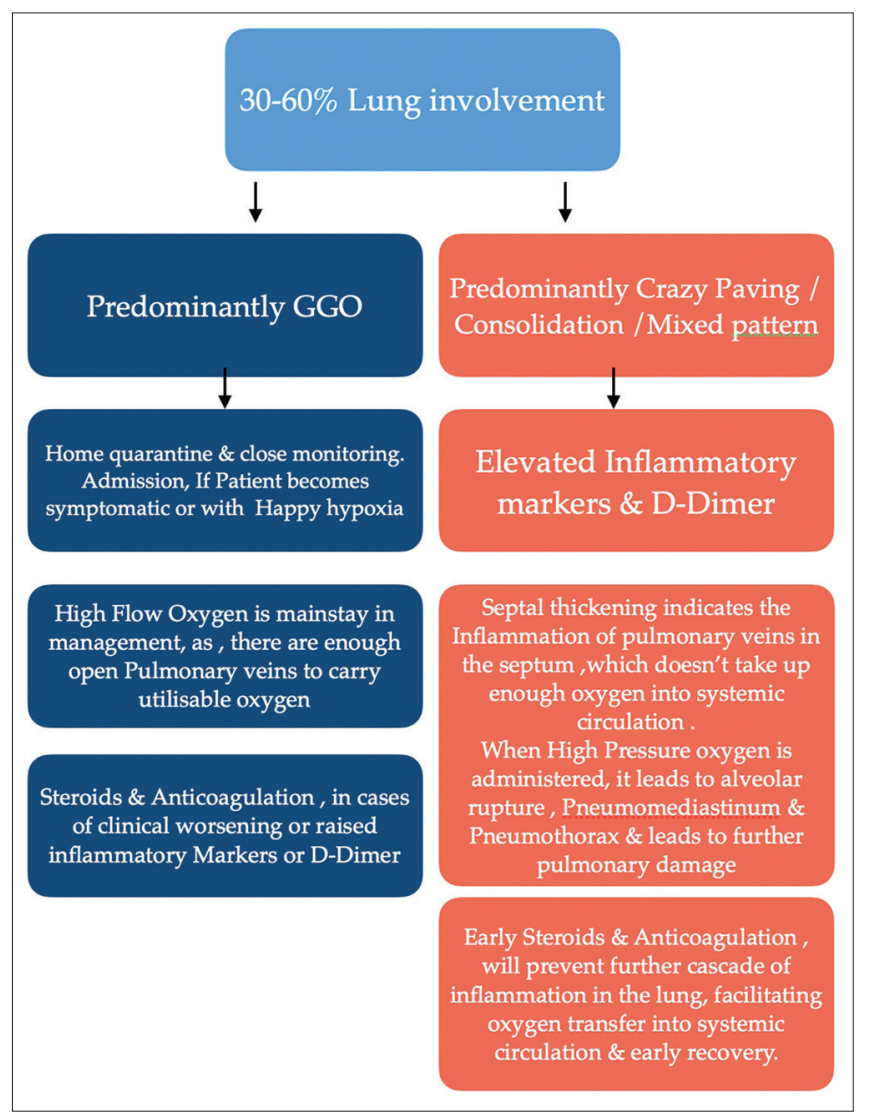

Chart 3: Patterns in cases with Less than 30-60\% Lung involvement on HRCT by Covid-19 with investigation and management protocol 
A significant proportion of these patients who had moderate disease by HRCT with ' $\mathrm{M}$ " or " $\mathrm{C}$ " pattern showed elevated Inflammatory markers and D-Dimer levels and showed excellent recovery when they were appropriately treated with steroids (dexamethasone) and Anticoagulants (therapeutic doses Low Molecular Weight Heparin). They do not progress to severe illness, as steroids and anticoagulants prevent the progressive inflammation of the lung parenchyma, thereby improving the oxygen delivery and absorption into systemic circulation. A large-size RCT (the RECOVERY trial) demonstrated that dexamethasone reduces deaths by one-third among critically ill COVID-19 patients. In the intervention group, 2,100 patients received dexamethasone $(6 \mathrm{mg} /$ day for 10 days) whereas in the control group patients $(n=4,300)$ received standard care for the disease. ${ }^{[18]}$ Because/COVID-19 patients have a higher incidence of venous thromboembolism and anticoagulant therapy is associated with reduced ICU mortality, it is suggested that patients should receive thromboprophylaxis. In the case of known thrombophilia or thrombosis, full therapeutic-intensity anticoagulation (e.g., enoxaparin $1 \mathrm{mg} / \mathrm{kg}$ twice daily) is indicated ${ }^{[19]} \mathrm{All}$ patients treated with steroids were monitored with serial blood sugar evaluation and in case of Hyperglycemia, treatment with insulin initiated, as and when required.

The patients with less than $<60 \%$ lung involvement are the ones that can be prevented from progression to severe disease. This will help decrease the burden on the health care workers and on the medical system in India, which are already overburdened by the disease. Moreover only $70 \%$ of the Covid affected patients show RT-PCR positive results and the turnover time for the result is longer these days, which makes $\mathrm{CT}$ a dependable tool in quick triaging and guiding the management of patients with Covid pneumonia.

\section{Conclusion}

In this Pandemic era, HRCT chest is a wonderful tool in triaging of patients for Home quarantine or Close monitoring with expectant management or Hospital Admission in cases of mild to moderate Lung involvement cases, along with clinical and biochemical evaluation. The Percentage of Pattern of Lung involvement by Covid on HRCT chest is important than the overall Severity score. The patients with Moderate Lung involvement by HRCT with predominantly Crazy paving or consolidation or mixed patterns showed elevated inflammatory markers and D-Dimer, in which cases, Steroid and Anticoagulants started early in the disease, showed improvement in symptoms, halting progression to severe disease, facilitating early recovery, decrease in depletion of finite resources, thereby can reduce the cost incurred and burden on the health care facilities across the world.

\section{Declaration of patient consent}

The authors certify that they have obtained all appropriate patient consent forms. In the form the patient(s) has/have given his/her/their consent for his/her/their images and other clinical information to be reported in the journal. The patients understand that their names and initials will not be published and due efforts will be made to conceal their identity, but anonymity cannot be guaranteed.

Financial support and sponsorship

Nil.

\section{Conflicts of interest}

There are no conflicts of interest.

\section{References}

1. Ai T, Yang Z, Hou H, Zhan C, Chen C, Lv W, et al (2020) Correlation of chest $\mathrm{CT}$ and RT-PCR testing in coronavirus disease 2019 (COVID-19) in China: A report of 1014 cases. Radiology 200642. 10.1148/radiol. 2020200642.

2. Hansell DM, Bankier AA, MacMahon H, McLoud TC, Müller NL, Remy J (2008) Fleischner society: Glossary of terms for thoracic imaging. Radiology 246:697-722. 10.1148/radiol. 2462070712.

3. Salehi S, Abedi A, Balakrishnan S, Gholamrezanezhad A (2020) Coronavirus disease 2019 (COVID-19): A systematic review of imaging findings in 919 patients. AJR Am J Roentgenol: 1-7. 10.2214/AJR.20.23034.

4. Ye Z, Zhang Y, Wang Y, Huang Z, Song B (2020) Chest CT manifestations of new coronavirus disease 2019 (COVID-19): A pictorial review. Eur Radiol. 10.1007/s00330-020-06801-0.

5. Prokop M, van Everdingen W, van Rees Vellinga T, Quarles van Ufford J, Stöger L, Beenen L, Geurts B, Gietema H, Krdzalic J, Schaefer-Prokop C, van Ginneken B, Brink M. CO-RADS - A categorical CT-assessment scheme for patients with suspected COVID-19: Definition and evaluation. Radiology 2020. doi: 10.1148/ radiol.2020201473.

6. Cascella M, Rajnik M, Cuomo A, Dulebohn SC, Napoli RD, et al. Features, Evaluation, and Treatment of Coronavirus (COVID-19) [Updated 2020 Aug 10]. In: StatPearls [Internet]. Treasure Island (FL): StatPearls Publishing; 2020 Jan-. Available from: https://www.ncbi.nlm.nih.gov/books/ NBK554776.

7. Clark A, Jit M, Warren-Gash C, Guthrie B, Wang HHX, Mercer SW, Sanderson C, McKee M, Troeger C, Ong KL, Checchi F, Perel P, Joseph S, Gibbs HP, Banerjee A, Eggo RM., Centre for the Mathematical Modelling of Infectious Diseases COVID-19 working group. Global, regional, and national estimates of the population at increased risk of severe COVID-19 due to underlying health conditions in 2020: A modelling study. Lancet Glob Health. 2020 Aug; 8(8):e1003-e1017.

8. Ciceri F, Beretta L, Scandroglio AM, Colombo S, Landoni G, Ruggeri A, Peccatori J, D'Angelo A, De Cobelli F, Rovere-Querini P, Tresoldi M, Dagna L, Zangrillo A. Microvascular COVID-19 lung vessels obstructive thromboinflammatory syndrome (MicroCLOTS): An atypical acute respiratory distress syndrome working hypothesis. Crit Care Resusc. 2020 Apr 15;22(2):95-97.

9. Flieder DB. Chapter-Normal Anatomy, Tissue Artifacts, and Incidental Structures. In: Zander DS, Farver CF. Pulmonary Pathology. (2017) ISBN: 9780323393089.

10. Itoh H, Murata K, Kenisha J, Nishimura K, Kitaichi M, Izumi T. Diffuse lung disease: Pathologic basis for the high-resolution 
computed tomography findings. (1993) Journal of thoracic imaging. 8(3):176-88. PubMed.

11. Heitzman ER, Markarian B, Berger I, Dailey E. The secondary pulmonary lobule: A practical concept for interpretation of chest radiographs. I. Roentgen anatomy of the normal secondary pulmonary lobule. (1969) Radiology. 93(3):507-12. doi: 10.1148/93.3.507-PubMed.

12. Webb WR. Thin-section CT of the secondary pulmonary lobule: Anatomy and the image--the 2004 Fleischner lecture. (2006) Radiology. 239(2): 322-38. doi: 10.1148/radiol. 2392041968-PubMed.

13. Tian S, Hu W, Niu L, Liu H, Xu H, Xiao SY. Pulmonary Pathology of Early-Phase 2019 Novel Coronavirus (COVID-19) Pneumonia in Two Patients With Lung Cancer. J Thorac Oncol. 2020 May; 15(5):700-704.

14. Zhang H, Zhou P, Wei $Y$, Yue H, Wang Y, Hu M, Zhang S, Cao T, Yang C, Li M, Guo G, Chen X, Chen Y, Lei M, Liu H, Zhao J, Peng P, Wang CY, Du R. Histopathologic Changes and SARS-CoV-2 Immunostaining in the Lung of a Patient With COVID-19. Ann. Intern. Med. 2020 May 05;172(9):629-632.

15. Menter T, Haslbauer JD, Nienhold R, Savic S, Hopfer H, Deigendesch N, Frank S, Turek D, Willi N, Pargger H, Bassetti S, Leuppi JD, Cathomas G, Tolnay M, Mertz KD, Tzankov A. Postmortem examination of COVID-19 patients reveals diffuse alveolar damage with severe capillary congestion and variegated findings in lungs and other organs suggesting vascular dysfunction. Histopathology. 2020 May 04.

16. Simpson S, Kay FU, Abbara S, Bhalla S, Chung JH, Chung M, et al. Radiological Society of North America Expert Consensus Statement on Reporting Chest CT Findings Related to COVID-19. Endorsed by the Society of Thoracic Radiology, the American College of Radiology, and RSNA. Radiology: Cardiothoracic Imaging 2020 2:2. https://doi.org /10.1148/ryct.2020200152.

17. Francone M, Iafrate F, Masci GM, Coco S, Cilia F, Manganaro L, Panebianco V, Andreoli C, Colaiacomo MC, Zingaropoli MA, Ciardi MR, Mastroianni CM, Pugliese F, Alessandri F, Turriziani O, Ricci P, Catalano C. Chest CT score in COVID-19 patients: Correlation with disease severity and short-term prognosis. Eur Radiol. 2020 Jul 4:1-10. doi: 10.1007/ s00330-020-07033-y. Epub ahead of print. PMID: 32623505; PMCID: PMC7334627.

18. Ledford H. Coronavirus breakthrough: Dexamethasone is first drug shown to save lives. Nature. 2020 Jun; 582(7813):469.

19. Kollias A, Kyriakoulis KG, Dimakakos E, Poulakou G, Stergiou GS, Syrigos K. Thromboembolic risk and anticoagulant therapy in COVID-19 patients: Emerging evidence and call for action. Br. J. Haematol. 2020 Jun; 189(5):846-847. 